\title{
Macrophage-Derived Chemokine Measurement
}

National Cancer Institute

\section{Source}

National Cancer Institute. Macrophage-Derived Chemokine Measurement. NCI

Thesaurus. Code C81956.

The determination of the amount of macrophage-derived chemokine present in a sample. 